\title{
ACARDIUS ACORMUS: UNA PRESENTACIÓN ATÍPICA EN EL EMBARAZO GEMELAR. REVISIÓN DE LA LITERATURA
}

\author{
Acardius acormus: an atypical presentation in twin \\ pregnancy. A literature review
}

María Mónica Cruz-Hernández*, Juan Luis Jaramillo-Valencia**, Natasha MejíaGarcía*, Jorge Hernán Gutiérrez-Marín, M.D.***, José Enrique Sanín-Blair, M.D.***

Recibido: junio 5/09 - Aceptado: noviembre 17/09

\section{RESUMEN}

Objetivo: presentar un caso de una gestación gemelar monocoriónica biamniótica con acardia fetal para revisar su diagnóstico y manejo.

Presentación del caso: se reportó el caso de un feto acardius acormus en un embarazo gemelar monocoriónico biamniótico diagnosticado a la semana $21+4$ días, por lo cual se realizó posterior seguimiento del caso.

Metodología: se revisó la historia clínica y los resultados de ayudas diagnósticas de la paciente. Posteriormente, se realizó una búsqueda bibliográfica en las bases electrónicas PubMed y SciELO.

Resultados: se llevó a cabo la revisión de la literatura con respecto a este caso poco frecuente y se encontraron 30 artículos relacionados. De éstos, se seleccionaron 15 en total: 9 artículos de revisión, 2 reportes de caso, 3 series de casos y 1 nota de epidemiología.

Conclusiones: la acardia fetal es una de las complicaciones poco frecuentes de la gestación gemelar

* Estudiantes de X Semestre de Medicina, Universidad Pontificia Bolivariana. Medellín (Colombia).

** Estudiante de XI Semestre de Medicina, Universidad Pontificia Bolivariana. Medellín (Colombia).

*** Especialista en Medicina Materno-Fetal. Profesor de la Universidad Pontificia Bolivariana. Coordinador de la Unidad de Alto Riesgo Obstétrico, Clínica Universitaria Bolivariana. Medellín (Colombia). Correo electrónico: saninblair@yahoo.com monocoriónica, cuya génesis y tasa de complicaciones se debe a las comunicaciones vasculares placentarias. El diagnóstico temprano y la vigilancia del caso presentado probablemente establecieron el buen desenlace perinatal.

Palabras clave: placenta, feto, complicaciones del embarazo, reporte de caso.

\section{SUMMARY}

Objective: presenting a case of a foetal arcadia occurring in monochorionic biamniotic twin pregnancy for reviewing its diagnosis and treatment. Case presentation: this is a case report of an acardius acormus foetus occurring in a monochorionic twin pregnancy diagnosed at week $21+4$ days and its clinical follow-up.

Methodology: the patient's clinical history and diagnostic test results were reviewed. This was followed by a literature search using PubMed and SciELO medical databases.

Results: a literature review was made regarding this atypical case; 30 interesting reports were found. Fifteen articles were selected: 9 review articles, 2 case reports, 3 case series and 1 epidemiology note.

Conclusions: foetal arcadia is one of the rare complications of monochorionic twin pregnancy and its complication rate is due to placental vascular 
communications. Early diagnosis and surveillance in this case probably led to a good perinatal outcome.

Key words: placenta, foetus, pregnancy complication, case report.

\section{INTRODUCCIÓN}

La acardia fetal, también conocida como secuencia de perfusión arterial gemelar retrógrada (TRAP, por sus siglas en inglés), es una complicación única de los embarazos gemelares monocoriónicos caracterizada por la ausencia del desarrollo del corazón en uno de los fetos a quien se le denomina gemelo receptor acárdico. Este feto es alimentado por medio de una anastomosis placentaria arterio-arterial de forma retrógrada por su gemelo (gemelo donante). ${ }^{1,2}$ Es una condición poco frecuente con una prevalencia de 1:35.000 embarazos y de 1:100 embarazos gemelares monocoriales. ${ }^{3-5}$ Es más frecuente en primigestantes, en fetos de sexo femenino y en pacientes sin historia de embarazos múltiples. ${ }^{6,7}$ Asimismo, y debido al espectro de complicaciones del feto donante, asociado, a su vez, con un espectro de malformaciones y anomalías, se ha observado que la mortalidad perinatal es reportada hasta en $55 \%$ de los casos. ${ }^{1}$

La fisiopatología de la enfermedad ha sido explicada por dos teorías:

1. Vasculatura placentaria anormal que lleva a una circulación reversa con alteración subsecuente del desarrollo cardíaco.

2. Embriogénesis cardíaca anormal como evento primario. $^{2}$

Del mismo modo, las diferentes publicaciones clasifican la acardia en 4 tipos:

Acardius acephalus: (es el más común [65\% de los casos]). El feto no presenta estructuras craneales o torácicas y usualmente las extremidades superiores se encuentran malformadas.

Acardius anceps: (22\% de frecuencia). El feto presenta algunas estructuras craneales y es el más parecido a la forma humana normal.

Acardius amorphus: (8\% de frecuencia). En este tipo, el feto está muy malformado, similar a un teratoma.
Acardius acormus: (5\% de frecuencia). El feto presenta mayor desarrollo en la cabeza que en el resto del cuerpo con poca o ausente formación de extremidades, siendo éste el fenotipo más raro. ${ }^{6,8-12}$

De otra parte, pese a ser una patología poco frecuente, el diagnóstico y el tratamiento oportunos de esta complicación tienen impacto en el desarrollo y desenlace del embarazo; por lo cual, es un reto para los profesionales de la salud hacer un enfoque integral de las mujeres con embarazo gemelar. Por tal motivo, el objetivo de este reporte es analizar los anteriores aspectos en el manejo de esta entidad por medio de la revisión de la literatura. Es importante aclarar que la paciente dio el consentimiento para la publicación del caso, respetando el principio de autonomía y privacidad de la misma.

\section{PRESENTACIÓN DEL CASO}

Mujer de 32 años, de raza negra, multigestante, con antecedente de dos cesáreas previas y preeclampsia, procedente de Apartadó (Antioquia), población ubicada en la zona noroccidental de Colombia. La paciente es referida a la unidad de medicina materno-fetal de la Clínica Universitaria Bolivariana (Institución de Tercer Nivel de complejidad para pacientes con aseguramiento contributivo y subsidiado) debido a que un ultrasonido obstétrico de rutina en la semana $20+5$ días reveló hallazgos sospechosos de anomalía discordante en uno de los fetos. Ante este hecho, se decidió realizar una ecografía de detalle anatómico y Doppler donde se evidenció una gestación gemelar monocorial biamniótica, existiendo un feto acárdico con secuencia TRAP (figuras 1 y 2). Debido a que el tamaño del feto acárdico era inferior a 70\% comparado con el gemelo donante y no existían (mediante Doppler y valoración ecocardiográfica funcional) signos de descompensación en este gemelo, se procedió a efectuar Doppler fetal y controles por alto riesgo obstétrico cada dos semanas.

Posteriormente, la paciente y el feto donante dan muestra de una evolución satisfactoria a la semana 35 de gestación cuando asiste a revisión por 
Figura 1. Doppler de la arteria umbilical única del feto acárdico que demuestra un flujo inverso (de la placenta al feto y no del feto a la placenta)

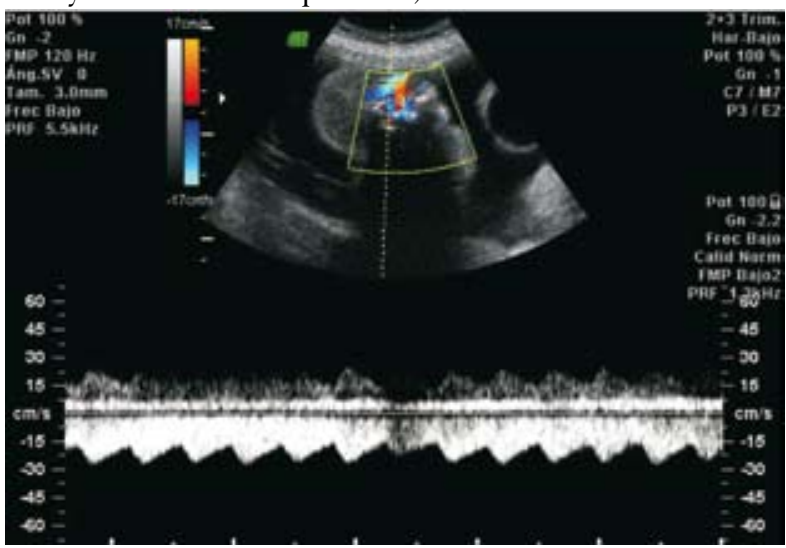

Figura 2. Se evidencia una masa amorfa mediante ultrasonido con arteria umbilical única. Gemelo receptor.

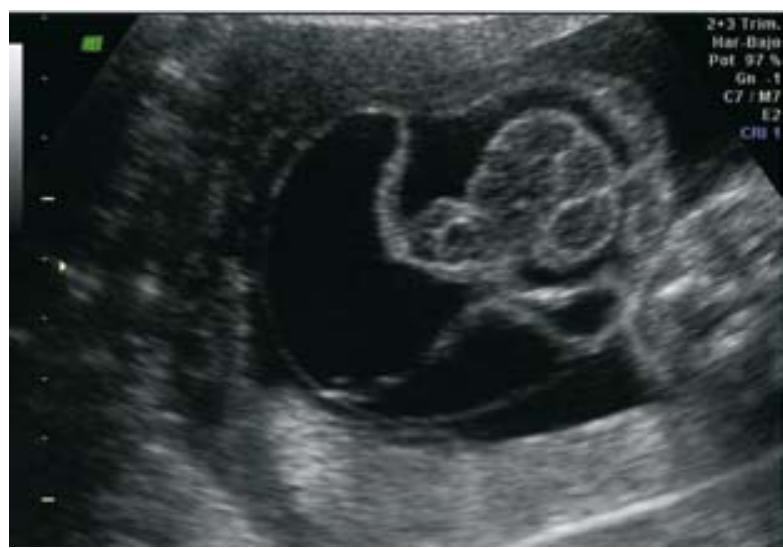

medicina materno-fetal, en donde es hospitalizada por presentar cefalea, epigastralgia, edema grado I en miembros inferiores y amenaza de parto pretérmino. Ante esto, se le practicó cesárea segmentaria el mismo día, en la cual se obtuvieron los fetos (figura 3). El gemelo donante presentó un peso de $2.550 \mathrm{~g}$, índice de APGAR 5 al primer minuto y 8 a los 5 minutos. La evolución neonatal no presentó alteraciones y la ecografía transfontanelar no evidenció anomalías estructurales neurológicas. El gemelo acárdico pesó $450 \mathrm{~g}$, dicho feto presentó poco desarrollo de extremidades superiores, inferiores y tronco con su porción cefálica más desarrollada. De acuerdo a estas características, éste último fue clasificado dentro de la variedad acardius acormus (figura 3).
Figura 3. Visión macroscópica de la placenta, los cordones y la anastomosis vascular. Se observa el feto acárdico con poco desarrollo de extremidades y tronco y porción cefálica de mayor tamaño.

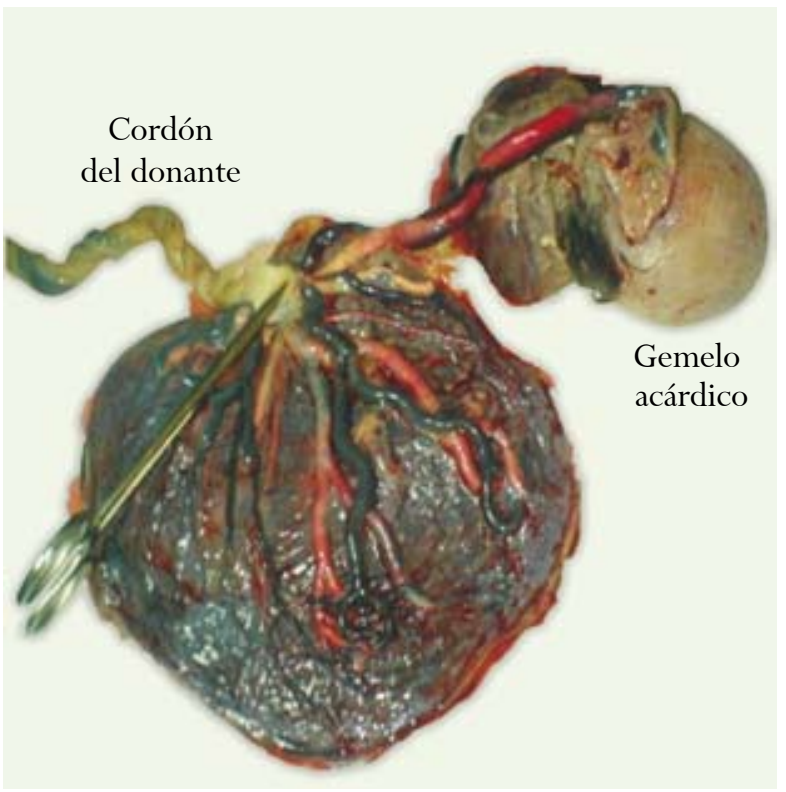

Por otro lado, la evolución postoperatoria de la paciente fue satisfactoria.

\section{METODOLOGÍA}

Se revisó la historia clínica y los resultados de ayudas diagnósticas de la paciente. A partir de lo anterior, se realizó una búsqueda bibliográfica en las bases electrónicas PubMed/Medline y SciELO mediante las palabras "sequence (TRAP)", "twin pregnancy", "acardiac fetus" y "acardius acormus".

\section{RESULTADOS}

Se llevó a cabo una revisión de la literatura con respecto a este caso poco frecuente y se encontraron 30 artículos de interés. De éstos se seleccionaron un total de 15: 9 artículos de revisión, 2 reportes de caso, 3 series de casos y 1 nota de epidemiología.

\section{DISCUSIÓN}

En este caso se presenta una rara variedad de la gestación gemelar monocorial mejor conocida como secuencia TRAP (Twin Reversed Arterial Perfusion), sobre la cual existen varias series de casos 
acerca de su adecuado diagnóstico y seguimiento. ${ }^{1-6}$ Es importante señalar que el diagnóstico de este trastorno se debe hacer en forma temprana antes del nacimiento (prenatal) por medio de una ecografía (a partir de la decimoprimera semana). Ahora bien, los hallazgos ecográficos característicos son: embarazo gemelar monocorial, flujo reverso por una arteria umbilical única y desarrollo discordante de uno de los gemelos con ausencia o poco desarrollo cardíaco. Por consiguiente, y a partir de estos hallazgos, se debe hacer diagnóstico diferencial con teratoma. ${ }^{6}$

El Doppler de arteria umbilical muestra el flujo retrógrado y sirve, junto con otras variables funcionales (ecocardiografía funcional y Doppler venoso), para realizar un seguimiento y definir el tratamiento o la intervención fetal a seguir. ${ }^{11,13-15}$ En el caso reportado, el Doppler fetal no evidenció signos de anemia fetal, polihidramnios, ni sobrecarga cardíaca; además, se comprobó que el feto acárdico presentó flujo inverso en arteria umbilical.

Teniendo en cuenta las características macroscópicas del feto, se concluyó que este caso hacía parte de la variedad acormus, lo cual le da mayor interés y relevancia por su escasa frecuencia. Aunque existen algunos reportes de caso en Colombia de secuencia TRAP, el presente es, a nuestro conocimiento, el primer caso reportado en el país de esta variedad de gemelo acárdico.

Por otro lado, el gemelo donante presentó maduración pulmonar tardía y su riesgo de fallecer in utero es alto debido a una insuficiencia cardíaca congestiva como consecuencia de la sobrecarga de flujo, además de otras complicaciones como: polihidramnios, anemia, parto prematuro e hidrops..$^{10,11}$ Debido a esto, se han propuesto alternativas de manejo para la secuencia TRAP, de tipo conservador o intervencionista quirúrgico, dirigido a disminuir la morbimortalidad del feto bomba y de la madre. Entre las intervenciones para lograr este objetivo se encuentran: administración de inotrópicos (digoxina) y AINE's (antiinflamatorios no esteroideos) en la madre para el control del polihidramnios, la insuficiencia cardíaca y para evitar el parto prema- turo, respectivamente. ${ }^{11}$ Otra opción terapéutica, es la realización de amniocentesis seriadas (amniodrenaje) para reducir el polihidramnios y así prolongar la gestación. ${ }^{12,13}$ También se ha realizado histerotomía selectiva para extraer el feto acárdico; no obstante, esta práctica ha entrado en desuso por las complicaciones materno-fetales que implica. ${ }^{10}$ Asimismo, en las ultimas décadas se han utilizado sustancias trombogénicas, alcohol absoluto, solución salina, ligadura del cordón umbilical, coagulación o termoablación con radiofrecuencia ${ }^{8}$ con el propósito de interrumpir el flujo sanguíneo del feto acárdico. Sin embargo, en nuestra experiencia, el método preferido y más eficaz es la realización de oclusión fetoscópica del cordón del receptor mediante coagulación con corriente bipolar.

De manera adicional, el manejo expectante es considerado, en la mayoría de los casos, paralelo a una estrecha vigilancia de la madre. Pese a esto, se ha observado muerte del feto bomba u otras complicaciones del mismo. ${ }^{1,2,8,11-15}$

\section{CONCLUSIÓN}

De acuerdo a las observaciones y a la revisión de la literatura, podemos concluir que la secuencia TRAP es una entidad relativamente rara que genera una gran morbimortalidad materno-fetal. Por este motivo, es importante hacer el diagnóstico prenatal para determinar su pronóstico y el tratamiento a seguir. En este caso, el resultado perinatal fue favorable con un seguimiento estricto y sin ninguna intervención quirúrgica.

\section{REFERENCIAS}

1. Tan TY, Sepúlveda W. Acardiac twin: a systematic review of minimally invasive treatment modalities. Ultrasound Obstet Gynecol 2003;22:409-19.

2. Sepúlveda W, Wong AE, Pons A, Gutiérrez J, Corral E. Secuencia de perfusión arterial reversa (gemelo acárdico): evaluación prenatal y tratamiento. Rev Chil Ultrasonog 2005;8:118-30.

3. Sepúlveda-Agudelo J. Secuencia de perfusión arterial inversa gemelar: revisión de la literatura. Rev Colomb Obstet Ginecol 2008;59:140-6. 
4. Rodeck C, Deans A, Jauniaux E. Thermocoagulation for the early treatment of pregnancy with an acardiac twin. N Eng J Med 1998;339:1293-5.

5. Brassard M, Fouron JC, Ledu L, Grignon A, Proulx F. Prognostic markers in twin pregnancies with an acardiac fetus. Obstet Gynecol 1999;94:409-14.

6. Napolitani FD, Schreiber I. The acardiac monster: a review of the world literature and presentation of 2 cases. Am J Obstet Gynecol 1960;80:582-9.

7. James WH. A note on the epidemiology of acardiac monster. Teratology 1977;16:211-6.

8. Weisz B, Peltz R, Chayen B, Oren M, Zalel Y, Achiron $\mathrm{R}$, et al. Tailored management of twin reversed arterial perfusion (TRAP) sequence. Ultrasound Obstet Gynecol 2004;23:451-5.

9. Chanoufi MB, Ben Temime R, Masmoudi A, Ounaïssa K, Jebnoun S, Abid W, et al. Clinical and anatomic features of acardiac twins. Med Princ Pract 2004;13:375-9.

10. Torres-Borrego J, Guzmán-Cabañas J, Arjona-Berral JE, Acosta-Collado A, Romanos-Lezcano A. Acardius acephalus: descripción de un caso. An Pediatr (Barc) 2000;53:346-9.

11. Galindo A, Puente JM, Benedicto M, Gutiérrez Larraya F. Gestaciones gemelares con feto acárdico: revisión a propósito de cinco casos. Actualidad Obstétrico Ginecológica 2001;13:287-90.

12. Romero M, Villalobos N, Ávila A, Fassolino C, López C. Acardia fetal en embarazo triple: reporte de un caso. Rev Obstet Ginecol Venez 2002;62:203-6.

13. Dashe JS, Fernández CO, Twickler DM. Utility of Doppler velocimetry in predicting outcome in twin reversed-arterial perfusion sequence. Am J Obstet Gynecol 2001;185:135-9.

14. Moore TR, Gale S, Benirschke K. Perinatal outcome of forty nine pregnancies complicated by acardiac twinning. Am J Obstet Gynecol 1990;163:907-12.

15. Sherer DM, Armstrong B, Shah YG, Metlay LA, Woods JR Jr. Prenatal sonographic diagnosis, Doppler velocimetry umbilical cord studies and subsequent management of an acardiac twin pregnancy. Obstet Gynecol 1989;74:472-5. 